\title{
Neural Evidence for Individual and Cultural Variability in the Social Comparison Effect
}

\author{
Pyungwon Kang, ${ }^{1,3}$ Yongsil Lee, ${ }^{1,2}$ Incheol Choi, ${ }^{4}$ and Hackjin Kim ${ }^{1,2}$ \\ ${ }^{1}$ Laboratory of Social and Decision Neuroscience, ${ }^{2}$ Department of Psychology, and ${ }^{3}$ Department of Brain and Cognitive Engineering, Korea University, \\ Seoul 136-701, South Korea, and ${ }^{4}$ Department of Psychology, Seoul National University, Seoul 151-742, South Korea
}

\begin{abstract}
Although several studies have investigated the neural mechanism of social comparison, it remains unclear whether and how cultural membership, particularly independent versus interdependent cultures, may differentially shape the neural processes underlying social comparison. In the present functional magnetic resonance imaging (fMRI) study, we examined the behaviors and neural response patterns of Korean (i.e., interdependent culture) and American (i.e., independent culture) participants while performing a financial gambling task simultaneously and independently with a partner. Upon seeing the partner's income, greater modulation of the activity in the ventral striatum (VS) and the ventromedial prefrontal cortex (vmPFC) by relative gain was observed in Korean than American participants, suggesting greater sensitivity of Koreans toward social comparison. The strength of functional connectivity between the VS and the vmPFC predicted individual variability in the degree to which participants' decisions were affected by relative incomes. Additional model-based fMRI analysis further confirmed the primary role of the vmPFC in biasing decisions based on relative incomes. In summary, the present study provides the first neural evidence for decision biases due to social comparison and their individual and cultural variations.
\end{abstract}

\section{Introduction}

Social comparison plays a pivotal role in human behavior (Festinger, 1954) that often produces less optimal outcomes. For example, research suggests that even with an identical payoff, people are less satisfied if they earn less than their colleagues or people in the same occupation in other firms (Clark and Oswald, 1996). Moreover, people tend to choose a lower paying job with the same salary as other employees than a higher paying job with a smaller salary compared with others (Tenbrunsel and Diekmann, 2002). As such, social comparison is an important determinant of the utility people experience from their choices. Recent neuroimaging studies have demonstrated that the human brain is also keenly attuned to social comparison information. For example, it was demonstrated that activity in the ventral striatum (VS), a well known reward-related brain region, was influenced by relative income (Fliessbach et al., 2007; Dvash et al., 2010), and increased at the misfortune of superior others (Takahashi et al., 2009).

It is now generally accepted that there exists a fundamental cultural difference in basic cognitive processes between Eastern and Western people (Nisbett et al., 2001). Although social com-

Received Oct. 18, 2012; revised Aug. 23, 2013; accepted Aug. 28, 2013.

Author contributions: Y.L. and H.K. designed research;P.K. performed research; P.K. analyzed data; P.K., I.C., and H.K. wrote the paper.

This research was supported by the Original Technology Research Program for Brain Science through the National Research Foundation (NRF) of Korea funded by the Ministry of Education, Science, and Technology (2012-0006587) and the NRF of Korea Grant, funded by the Korean Government (NRF-2011-327-H00038). We thank Hyoyoung Kim and Haehwang Lee for technical assistance.

Correspondence should be addressed to Hackjin Kim, Department of Psychology, Korea University, 145 Anam-ro, Sungbuk-Ku, Seoul, South Korea, 136-701. E-mail: hackjinkim@korea.ac.kr.

DOI:10.1523/JNEUROSCI.5084-12.2013

Copyright $\odot 2013$ the authors $\quad 0270-6474 / 13 / 3316200-09 \$ 15.00 / 0$ parison is commonly observed in every society (Gilbert et al., 1995), there is also growing evidence supporting the role of culture in modulating the degree of social comparison (Chung and Mallery, 1999; Gibbons and Buunk, 1999; White and Lehman, 2005). Specifically, individuals in interdependent cultures that stress relationships with others (e.g., East Asia) are more likely to seek social comparison than individuals in independent cultures that emphasize one's internal feelings and thoughts (e.g., North America). For example, self-reported collectivism is positively correlated with the degree of social comparison in general (Chung and Mallery, 1999), and interpersonal orientation is positively related to interest in social comparison (Gibbons and Buunk, 1999). In addition, White and Lehman (2005) showed that Asian (compared with European) Canadians sought more social comparison information and were more willing to check other students' scores following an intellectual test. Meanwhile, given recent neuroimaging evidence for cultural differences between East Asian and Western participants (Zhu et al., 2007; Chiao et al., 2009; B. Kim et al., 2012), we also aimed to examine cultural modulation of the neural activity sensitive to social comparison. Specifically, we expected that financial decisions would be more affected by relative income in Korean than American participants, and that this cultural difference would be mirrored in reward-related brain regions such as the nucleus accumbens and the orbitofrontal cortex.

Despite accumulating neural evidence for social comparison, however, no study has investigated the neural activity closely tied to actual decisions influenced by others, particularly on a trialby-trial basis. Matching neural activity with decisions would be critical for revealing the neural substrates responsible for decisions and accurately predicting individual variability in social 


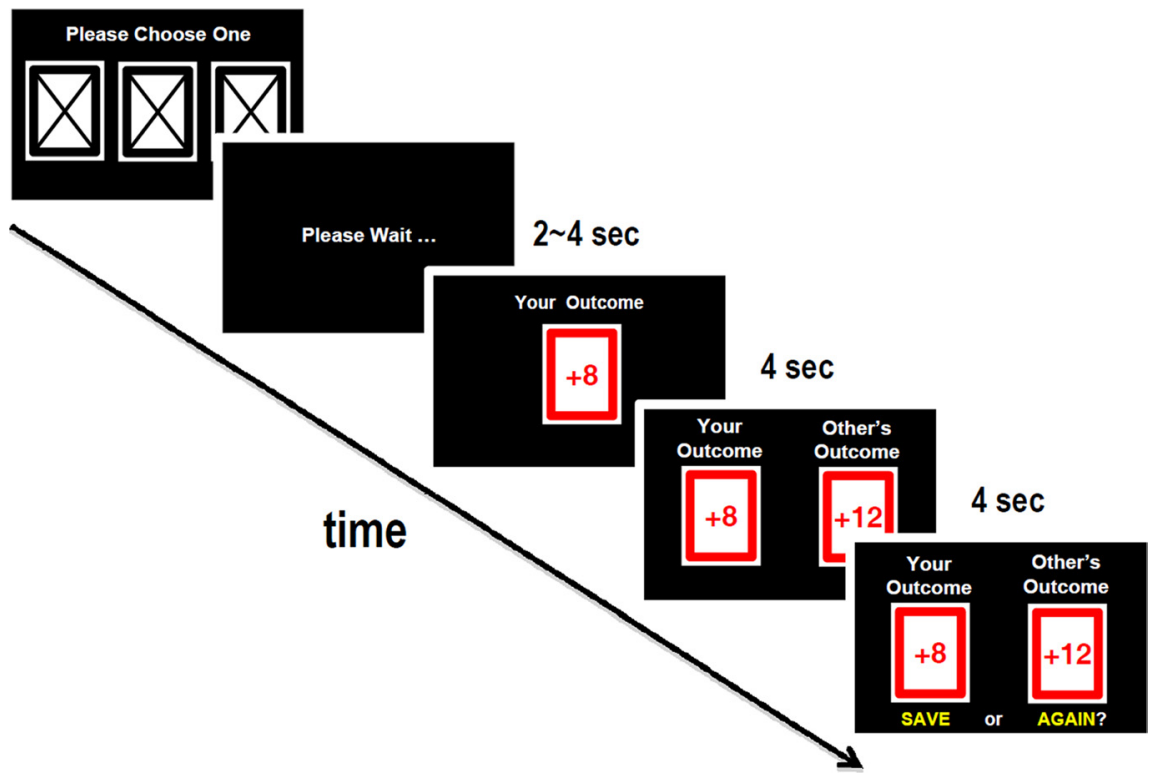

Figure 1. A diagram of a typical game trial. Each trial started with the presentation of three unrevealed cards; the participant is then asked to choose one of them. Two to four seconds later, the monetary outcome for the participant (AI) is displayed. Four seconds later, monetary outcomes for both the participant and the partner are displayed; another four seconds later, the participant is forced to choose whether to save the results or repeat the trial later.

comparison. Thus, we aimed to reveal that the neural circuitry sensitive to relative income is associated with individual variability in financial decision making affected by social comparison as well as cultural variation.

\section{Materials and Methods}

Participants. Eleven Koreans (age $44.00 \pm 4.16$ years, all female) and 11 Americans (age $41.18 \pm 5.64$ years, all female) participated in the experiment. The American participants were living in Korea when they participated in this experiment, but none of them could speak Korean or had been in Korea longer than 2 years. All participants were carefully screened for their income levels before recruitment; only those who reported mid-range income levels were included in the study. Korean and American participants were matched on the basis of age, education level, and family status. Participants were all right-handed, with normal or corrected-to-normal vision and no prior history of neurological or psychiatric disease. Informed consent was obtained from all the participants, and the study was approved by the institutional review board of Korea University.

Task. To elicit social comparison, we used a card game (Fig. 1) modified from a game-of-chance task used in a previous study (Dvash et al., 2010). Participants were instructed to play a card game with another participant who was ostensibly present in a separate room. Each cardgame trial began with the display of three unrevealed cards. Participants were instructed to choose one card knowing that their partner was also going to choose one, independently. Two to four seconds after choosing a card, participants saw their own outcome; $4 \mathrm{~s}$ later, their income and their partner's income were shown simultaneously. Finally, participants were asked to decide whether to keep the result of the trial (i.e., "save"), or to repeat the trial later (i.e., "again"). They were told that the outcome of the game would be determined by chance. The two players' games were performed independently so that their outcomes and decisions would not affect their partner's outcomes, and vice versa. That is, the participants could see only the outcomes of their partners' card choices-they were not informed which card was chosen by their partners or whether they chose save or again. The outcome of each card game trial was presented as a point score ranging from -12 to +12 by increments of 4 ; participants were told that each point score was comparable to approximately one dime (or KRW 100). Unknown to the participants, we presented the outcomes of -12 and +12 points only to the partners, not the participants, to achieve an equal variability of relative income levels (i.e., $-4,-2,0,2,4)$ for any given absolute income (AI) level. This type of design also allowed us to minimize correlation between the parameters of AI and relative income (RI), which was critical for the purpose of the present study. One potential drawback might be that we ended up with a greater variability of outcomes for partners than for participants, although the mean outcome levels were kept identical for both groups. In addition to our effort to orthogonalize the parameters of AI with respect to those of RI, we delayed the onset of outcome for the partner compared with that for the participant; this further allowed us to prevent potential multicollinearity issues between the parameters of AI and RI in the general linear models (GLMs).

The outcomes for participants and their partners were predetermined by the experimenter to control the number of trials for each absolute and relative income level, but the order of trials was pseudorandomly determined for each participant. There were five levels of AI and five levels of RI; each combination of the two factors was repeated twice, resulting in a total of 50 game trials. Participants were told that the sum of the outcomes of all the trials would be added to or subtracted from their initial endowments.

Before the functional magnetic resonance imaging (fMRI) experiment, each participant was provided detailed instructions for the game by an instructor who was a well trained native speaker of the participant's language. After completing a total of 50 game trials $(\sim 20 \mathrm{~min})$, participants were debriefed and compensated 25,000-30,000 KRW $(\approx 25$ USD) for participation.

fMRI data acquisition. All neuroimaging was performed by a 3 T MRI scanner (MAGNETOM Trio, A Tim System; Siemens Medical Solutions) with a 12-channel head coil. We acquired high-resolution anatomical images $\left(\mathrm{TR}=1900 \mathrm{~ms}\right.$; $\mathrm{TE}=2.52 \mathrm{~ms}$; flip angle $=9^{\circ}, 1 \times 1 \times 1 \mathrm{~mm}$ in-plane resolution; $256 \times 256$ matrix size) and gradient echo-planar images (EPI) with blood oxygenation level-dependent contrast (TR = $2000 \mathrm{~ms} ; \mathrm{TE}=30 \mathrm{~ms}$; flip angle $=90^{\circ} ; 3 \times 3 \times 4 \mathrm{~mm}$ in-plane resolution; $64 \times 64$ matrix size; 33 slices with no gap). We used an oblique acquisition sequence tilted at $30^{\circ}$ from the AC-PC line to increase the signal from the orbitofrontal cortex (Deichmann et al., 2003). E-prime 2.0 software (Psychology Software Tools) was used for implementing the experimental task.

fMRI data analysis. Neuroimaging data were preprocessed and analyzed using Statistical Parametric Mapping 8 (SPM8; the Wellcome Trust Centre for Neuroimaging, University College London, UK). Before the preprocessing of fMRI data, the first five volumes were discarded to allow the MR signal to reach steady-state equilibrium. Images were realigned to the first volume of each session to correct motion using the six headmotion parameters, and spatially normalized to the standard Montreal Neurological Institute EPI template, then smoothed with an isotropic Gaussian kernel with $6 \mathrm{~mm}$ full-width at half-maximum (FWHM).

We estimated three GLMs to analyze the fMRI data. All three models commonly included the regressors for the onset times of participants' save and again decisions, six estimated head-motion parameters, and a constant. All regressors of interest were convolved with a standard hemodynamic response function.

To identify regions of interest showing activity positively correlated with income levels, we estimated the first GLM containing the following regressors as parametric modulators: $\mathrm{AI}$ at the onset of the first outcome events, and RI and partner's AI at the onset of the second outcome events. Then, we conducted a group analysis of one-sample $t$ tests for the main effects of AI and RI combining individual contrast maps that showed correlation with the parameters of $\mathrm{AI}$ and RI. 
The second GLM was estimated to investigate the interaction effect of income type, income level, and culture as core factors modulating neural activity. This model contained the following regressors: absolute loss $(-8,-4)$, absolute zero $(0)$, absolute gain $(4,8)$, relative loss $(-4,-2)$, relative zero $(0)$, and relative gain $(2,4)$. In the second-level group analysis, we performed a $2($ Korean, American $) \times 2(\mathrm{AI}, \mathrm{RI}) \times 3$ (loss, zero, gain) mixed ANOVA.

The third GLM was generated to investigate the neural mechanisms involved in biasing decisions due to RI on a trial-by-trial basis. For this analysis, we estimated optimal sigmoid function best fitted to the subjects' decisions as a function of absolute income. To compute the degree of individuals' decision bias attributable to RI, model estimation errors (MEE) were calculated by subtracting an estimated decision probability $f\left(x_{i}\right)$ for a given trial $i$ with $\mathrm{AI} x$ from a decision $D_{i}$ on a trial-by-trial basis (see the equations below). Our rationale for the MEE analysis could be best conveyed by comparing two exemplary trials: one positive absolute income trial $\mathrm{A}(\mathrm{Me}:+8$, Other: +12 ) and a negative absolute income trial $\mathrm{B}$ (Me: -4 , Other: 0 ). Both trials A and B have the same payoff difference (or relative income level: RI), but the amount of bias in the decision would not be the same because the likelihood of a save outcome is higher for trial A compared with trial B due to the different AI level. Given that this likelihood also varies across individuals, it was necessary to first estimate each individual's decision likelihood as a function of AI level and then compute the decision bias from the estimated likelihood based on the actual decisions on a trial-by-trial basis.

$$
\begin{gathered}
f\left(x_{i}\right)=\frac{1}{e^{a(b-x)}+1} \\
\operatorname{MEE}(i)=D_{i}-f\left(x_{i}\right)\left\{\begin{array}{l}
D_{i}=1: \text { (save) } \\
D_{i}=0: \text { (again) }
\end{array}\right\} .
\end{gathered}
$$

Given that save is 1 and again is zero, MEEs greater than zero indicated a positive decision bias (i.e., choosing save), whereas MEEs less than zero indicated a negative decision bias (i.e., choosing again). Further, the absolute values of MEEs indicated the degree of decision bias in a direction determined by the associated signs. For the third GLM analysis, both $f\left(x_{i}\right)$ and $\operatorname{MEE}(i)$ were added as parametric modulators to the regressors of the decision events. For this analysis, we calculated individuals' regression coefficient maps for each parametric modulator; the resulting maps were used for one-sample $t$ tests.

To identify the neural structures communicating with the VS, we performed two psychophysiological interaction (PPI) functional connectivity analyses (Friston et al., 1997). PPI analysis assesses temporal correlations between brain regions and their dependencies on experimental conditions; thus, it can be used to investigate functional coupling between two brain regions modulated by experimental conditions. For our PPI analyses, we extracted a deconvolved time series (Gitelman et al., 2003) of the VS from each subject that showed significant correlations with AI (see GLM 1). For the first PPI analysis, individual PPI GLMs contained three regressors: the deconvolved time series data from the VS; a condition vector with 1 and -1 for relative gain and loss trials, respectively; and the product of the two regressors, that is, the PPI term. For the second PPI analysis, all other procedures were identical to the first PPI analysis, but the condition vector for relative gain and loss trials was replaced with the condition vector for relative gain and absolute gain trials. Each participant's coefficient image of the interaction term from the PPI analysis was then entered into the random-effects group analysis.

Determining statistical significance. To correct for multiple comparisons, we conducted a 10,000-iteration Monte Carlo simulation by using AlphaSim in AFNI software, which generates volumetric cluster sizes (Cox, 1996; Table 1). As criteria inputs to AlphaSim, we entered $p=$ 0.001 for individual voxel threshold; $9.18,8.96$, and $8.80 \mathrm{~mm}$ for smoothness (determined via $3 \mathrm{dFWHM}$ in AFNI); and $2 \times 2 \times 2$ for voxel size. For the analysis with the parameter of absolute income, we restricted the AlphaSim search volumes to spheres with a $10 \mathrm{~mm}$ radius $\left(\sim 4200 \mathrm{~mm}^{3}\right)$ for VS based on the coordinates reported in a previous neuroimaging study on social comparison (Fliessbach et al., 2007). For detecting significant activation clusters within the ventromedial prefrontal cortex

\begin{tabular}{|c|c|c|c|c|}
\hline \multirow[b]{2}{*}{ Brain region } & \multirow[b]{2}{*}{$Z$} & \multicolumn{3}{|c|}{ Peak in MNI } \\
\hline & & $x$ & $y$ & $z$ \\
\hline \multicolumn{5}{|l|}{ Correlation with absolute income } \\
\hline \multirow[t]{2}{*}{ Paracentral lobule (R) } & 4.86 & 2 & -32 & 54 \\
\hline & 4.29 & 6 & -36 & 60 \\
\hline \multirow[t]{4}{*}{ Lingual gyrus (L) } & 4.69 & -20 & -88 & -18 \\
\hline & 3.96 & -10 & -86 & -6 \\
\hline & 3.89 & -22 & -76 & -10 \\
\hline & 4.54 & 6 & -90 & 32 \\
\hline Postcentral gyrus (R) & 4.37 & 42 & -22 & 50 \\
\hline Precentral gyrus (R) & 4.02 & 44 & -16 & 44 \\
\hline Caudate nucleus (R) & 4.31 & 12 & 10 & -12 \\
\hline \multicolumn{5}{|l|}{ Correlation with relative income } \\
\hline \multirow[t]{5}{*}{ Middle frontal gyrus (L) } & 4.84 & -36 & 46 & 4 \\
\hline & 4.79 & -40 & 42 & 28 \\
\hline & 3.85 & -32 & 38 & 30 \\
\hline & 4.50 & -30 & 36 & 42 \\
\hline & 3.84 & -28 & 28 & 42 \\
\hline \multirow[t]{2}{*}{ Inferior parietal lobule (L) } & 4.33 & -56 & -32 & 44 \\
\hline & 4.20 & -62 & -42 & 40 \\
\hline \multicolumn{5}{|c|}{ Culture $X$ income type $X$ income level } \\
\hline vmPFC & 3.57 & 2 & 30 & 0 \\
\hline Medial orbitofrontal cortex* & 3.11 & 2 & 56 & -6 \\
\hline \multicolumn{5}{|c|}{$\begin{array}{l}\text { PPI with VS in the context of relative gain against } \\
\text { relative loss }\end{array}$} \\
\hline vmPFC & 3.52 & 6 & 38 & -2 \\
\hline \multicolumn{5}{|c|}{$\begin{array}{l}\text { PPI with VS in the context of relative loss against } \\
\text { relative gain }\end{array}$} \\
\hline Insula (R) & 4.01 & 48 & -6 & 12 \\
\hline \multirow[t]{2}{*}{ Fusiform gyrus (R) } & 3.63 & 40 & -44 & -12 \\
\hline & 3.47 & 38 & -32 & -20 \\
\hline \multicolumn{5}{|l|}{ Positive correlation with MEE } \\
\hline \multirow[t]{3}{*}{ Postcentral gyrus (R) } & 3.94 & 34 & -32 & 58 \\
\hline & 3.77 & 42 & -28 & 64 \\
\hline & 3.62 & 16 & 36 & -8 \\
\hline \multicolumn{5}{|l|}{ Negative correlation with MEE } \\
\hline Insular (R) & 4.28 & 34 & 18 & 14 \\
\hline
\end{tabular}

Table 1. Brain regions exceeding the threshold determined by AlphaSim

All are significant at $p<0.05$ (corrected) except $\left(^{*}\right)$.

(vmPFC), bilateral insula, and the whole brain, we restricted the search volumes to $\sim 11,000 \mathrm{~mm}^{3}, 17,300 \mathrm{~mm}^{3}$, and $672,900 \mathrm{~mm}^{3}$, respectively, according to the volume sizes reported in a previous study (Kennedy et al., 1998). The cluster extent to obtain $\alpha<0.05$ was $4,13,18$, and 80 voxels for VS, vmPFC, insula, and whole brain, respectively.

\section{Results}

\section{Behavioral results}

To characterize individual differences in social comparison tendency, we performed a logistic regression analysis to estimate the contributions of AI and RI to the probability of a save choice in each participant. We found that one participant (Korean) had made no again decisions and two others (one Korean and one American) showed excessively high coefficients for RI (i.e., over 3 SDs above the mean), mainly due to a misfit problem. As it was possible that these participants' extraordinary decision tendencies may have originated from unexpected factors rather than AI or RI, we excluded their data from the behavioral and neuroimaging analyses using the $\beta$ coefficients of logistic regression.

Then, we used the $\beta$ coefficients for AI and RI from the individual logistic regression analyses to carry out a two-way ANOVA with cultural membership (Korean, American) and income type (AI, RI) as factors. This analysis also yielded a marginally significant interaction between cultural membership and income type $\left(F_{(1,18)}=3.85, p=0.065\right.$; Fig. 2$)$. To reconfirm the cultural difference in social comparison-seeking behavior in a different way, a repeated-measures ANOVA with cultural membership 


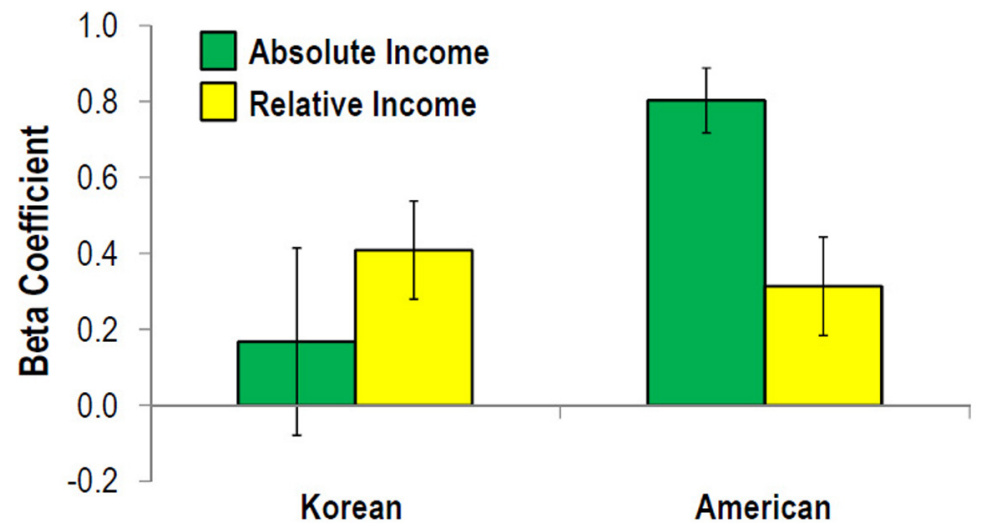

Figure 2. Behavioral results. A mixed ANOVA with $\beta$ coefficients from logistic regression analysis showed that the American participants' financial choices were more sensitive to absolute than relative income, compared to the Koreans.
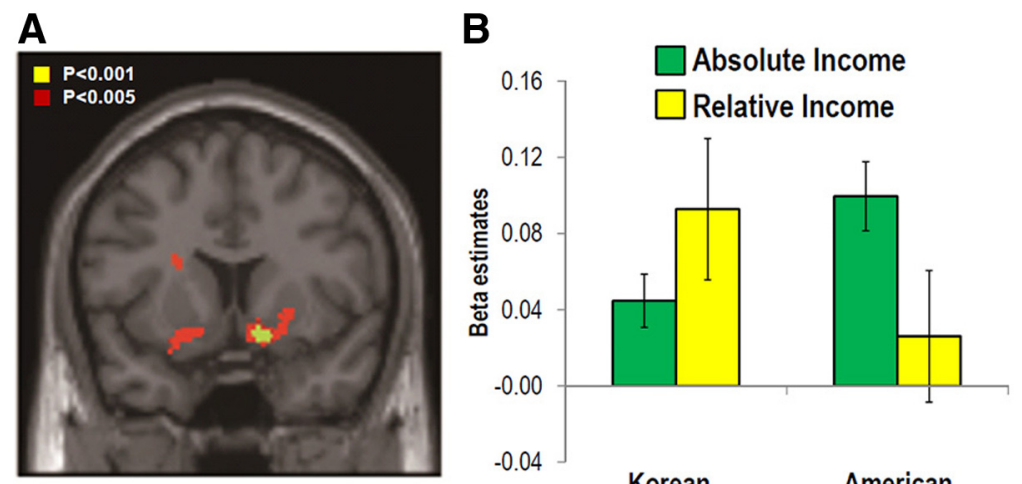

\section{Korean American}

Figure 3. A, A significant correlation with Al was found in the right VS $(x=12, y=10, z=-12)$. B , Parameter estimates of the peak voxel in the VS correlating with Al showed a significant interaction effect of income type (Al, RI) $\times$ cultural membership (Korean, American).

(Korean, American) as between-subjects variable and income type (AI, RI) and income level (five levels) as within-subjects variables was performed on the save decision. This analysis revealed a significant three-way interaction $\left(F_{(4,68)}=3.05, p=\right.$ $0.023)$. When we conducted the same ANOVA performed above including the three outliers to the behavioral data, a marginally significant three-way interaction was found $\left(F_{(4,80)}=1.74, p=\right.$ $0.15)$.

Further, a significant linear $\left(F_{(1,20)}=5.39, p=0.03\right)$ but not quadratic $\left(F_{(1,20)}=2.07, p=0.16\right)$ trend was found in the probability to choose save as a function of relative income. We also found that American and Korean participants showed significant linear trends over income level in all conditions (AI: American, $F_{(1,9)}=27.34, p=$ 0.001 , Korean, $F_{(1,8)}=5.17, p=0.053$; RI: American, $F_{(1,9)}=6.84$, $p=0.028$, Korean, $\left.F_{(1,8)}=14.66, p=0.005\right)$.

In sum, these findings indicate that Korean and American participants differed in terms of the degree to which their financial decisions were affected by absolute and relative financial outcomes. That is, American participants were more sensitive to AI than RI, compared with Korean participants.

\section{Neuroimaging results}

Region of interest analysis

Our first analysis focused on replicating previous studies and identifying a reward-sensitive brain region, the VS. Consistent with past research, the first analysis using the parameter of AI revealed VS $(x=12, y=10, z=-12, Z=4.31, p<0.05$, corrected) whose activity showed a significant correlation with AI (Fig. 3A).

We then investigated whether activity in the localized VS was modulated by participants' cultural membership, by carrying out a repeated-measures ANOVA with income type (2: gain and loss) $\times$ cultural membership (2: American and Korean) as factors, and with the $\beta$ estimates for the parametric regressors of AI and RI as dependent variables. As expected, we found a significant two-way interaction effect of income type $\times$ cultural member$\operatorname{ship}\left(F_{(1,20)}=5.13, p=0.035\right.$; Fig. $\left.3 B\right)$. More specifically, the results indicated that VS activity was modulated by AI rather than RI in the American participants $\left(t_{(10)}=1.82, p=0.045\right.$, one-tailed), whereas the opposite pattern was true in the Korean participants $\left(t_{(10)}=1.36, p=\right.$ 0.102 , one-tailed); this provided neural evidence supporting the behavioral difference observed in social comparison between American and Korean participants.

Voxelwise whole-brain analysis

In addition to the region of interest (ROI) analysis, we used a whole-brain search for other brain regions that are sensitive to cultural modulation of relative incomes, and performed a three-way factorial second-level analysis using income type (AI, RI), income level (gain, zero, loss), and cultural membership (Korean, American) as factors. This analysis revealed a prominent cluster within the vmPFC showing a significant three-way interaction effect ( $x=2, y=30, z=0, Z=3.57, p<0.05$, corrected). In this region, post hoc tests revealed that the American participants showed higher levels of activity in response to absolute gain $(\mathrm{M}=$ $0.69, \mathrm{SE}=0.35)$ versus loss $(\mathrm{M}=-0.48, \mathrm{SE}=0.27, p=0.001)$ but not to relative gain $(\mathrm{M}=0.06, \mathrm{SE}=0.22)$ versus loss $(\mathrm{M}=$ $0.14, \mathrm{SE}=0.20, p=0.71$, whereas the Korean participants exhibited increased levels of activity in response to relative gain $(\mathrm{M}=0.27, \mathrm{SE}=0.22)$ versus loss $(\mathrm{M}=-0.31, \mathrm{SE}=0.20, p=$ $0.015)$ but not to absolute gain $(\mathrm{M}=0.18, \mathrm{SE}=0.35)$ versus loss $(\mathrm{M}=0.32, \mathrm{SE}=0.27, p=0.449$; Fig. $4 B, C)$. The same analysis also revealed a significant linear trend of absolute income level for the American participants, $F_{(1,10)}=10.94, p=0.008$, but not for the Korean participants, $F_{(1,10)}=0.37, p=0.55$. On the contrary, a significant linear trend of relative income level was observed only for the Korean participants $\left(F_{(1,10)}=7.71, p=0.02\right)$, not the American participants $\left(F_{(1,10)}=0.12, p=0.72\right)$. A similar ANOVA on the VS shown in Figure 3 revealed a significant linear $\left(F_{(1,20)}=5.32, p=0.03\right)$, but not quadratic trend $\left(F_{(1,20)}=0.31\right.$, $p=0.49)$ over relative income level. It also revealed that the American and Korean participants showed significant linear trends over income level of AI (American: $F_{(1,10)}=39.84, p=$ 0.0001 ; Korean: $\left.F_{(1,10)}=9.12, p=0.013\right)$, but only the Korean participants showed linearly increasing activation levels of VS against RI (American: $F_{(1,10)}=0.64, p=0.44$; Korean: $F_{(1,10)}=$ $6.63, p=0.028)$. 


\section{PPI analysis}

Numerous previous reports have indicated a close relationship between the VS and the vmPFC, both structurally and functionally (Haber et al., 2006; Di Martino et al., 2008; Haber and Knutson, 2010). Therefore, keeping in mind the observation that the activation of both the VS and VmPFC was modulated by RI, we decided to test whether RI could also modulate the functional connectivity between the VS found in the ROI analysis and the vmPFC, by conducting a PPI analysis (Friston et al., 1997) with the peak voxel of the VS from the ROI analysis as a seed region. Considering that the probability of repeating correlated linearly with the relative payoff difference, we predicted that the communication between the two structures would also be modulated by relative payoff difference, that is, between relative gain and loss.

This analysis revealed a significant PPI interaction effect in the vmPFC $(x=6$, $y=38, z=-2, Z=3.52, p<0.05$, corrected; Fig. $5 A$ ), which means that functional connectivity between the VS and the vmPFC was stronger during the relative gain versus relative loss conditions. In addition, a negative functional connectivity between the VS and the right insula $(x=48$, $y=-6, z=12, Z=4.01, p<0.05$, corrected) was observed during the relative gain versus relative loss conditions. There was no such functional connectivity during the absolute gain (AG) versus absolute loss $(\mathrm{AL})$ conditions between the VS and the vmPFC, and also between the VS and the right insula.

To examine the communications in more detail, we also computed correlations between the time series of the VS and those for the vmPFC separately for each condition (i.e., relative gain (RG) and the relative equal (RE) and relative loss (RL) conditions). As shown in Figure $5 B$, the functional connectivity between the VS and the vmPFC was modulated linearly by the relative payoff difference $\left(F_{(2,40)}=3.52, p=0.039\right)$, consistent with the behavioral data. We also investigated whether this functional communication between the VS and the vmPFC was specific to the relative gain condition by running a similar PPI analysis with the psychological contrast variable of RG and AG trials. This analysis revealed a cluster in the vmPFC $(x=8, y=36, z=-2, Z=2.79)$ almost identical to that from the prior PPI analysis with a lenient threshold ( $p=0.003$, uncorrected). This finding further confirmed the hypothesis that the functional communication between the VS and vmPFC is most significantly modulated during the relative gain condition.

Naturally, one might also question whether the observed functional coupling between the VS and the vmPFC can predict the degree to which individuals' financial decisions were influenced by RI. To answer this question, we examined the relationship between the $\beta$ coefficients of RI attained from the logistic regression analysis and the $\beta$ estimates of the PPI term in the vmPFC. This revealed a significant correlation $(r=0.48, p=$ 0.037; Fig. $5 C$ ), suggesting that the strength of the functional coupling between the VS and the vmPFC is critical for a behavioral manifestation of social comparison. When the correlation was examined separately for each of the three RI levels, we found that
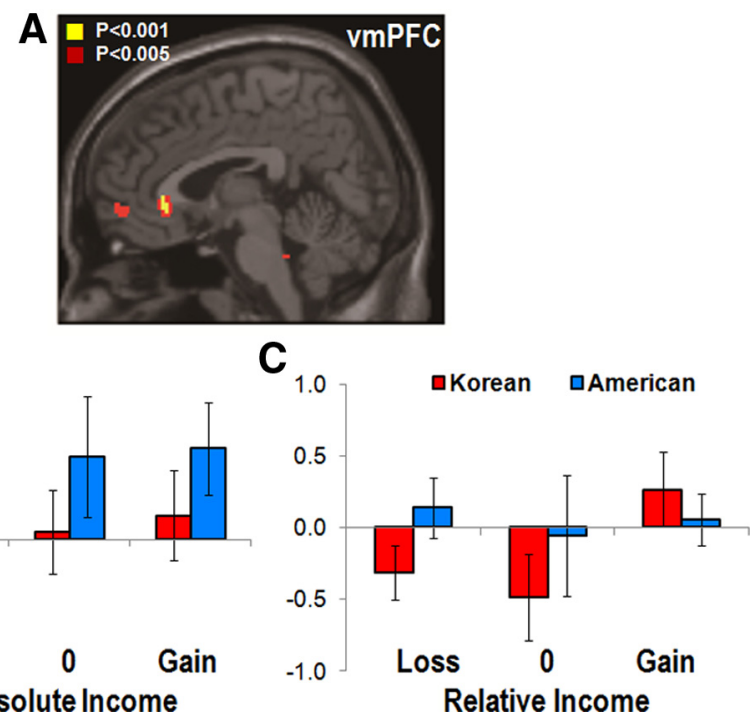

Figure 4. A three-way factorial ANOVA. $A, A$ three-way interaction of income type $\times$ income level $\times$ cultural memberwhereas the opposite pattern was observed in the Korean participants (C). The medial orbitofrontal cortex showed a similar

individual variability in social comparison-seeking propensity was predicted by the communication between the VS and the vmPFC significantly during RL $(r=0.50, p=0.03)$ and marginally during RG condition $(r=0.33, p=0.17)$, but not during the RE $(r=0.08$, $p=0.73$ ) condition. In addition, we performed the same analysis using $\mathrm{AI}$ and found that the correlation coefficients of the VS and vmPFC were not associated with participants' decisions (AG: $r=$ -0.16 , absolute equal (AE): $r=-0.21, \mathrm{AL}: r=-0.31$, all $p s>0.05)$.

For the right insula, its functional coupling with the VS did not predict the individual variability in social comparison $(r=$ $0.02, p=0.93)$.

\section{Neural signals for decision bias due to social comparison}

Based on our recent observation that orbitofrontal cortical activity encodes signals associated with decision bias due to social information (H. Kim et al., 2012), we specifically aimed to identify neural structures encoding signals for decision biases caused by social comparison. To determine the amount of decision bias attributable to RI on a trial-by-trial basis, we first computed subject-specific decision probability models by estimating the optimal sigmoid functions that best fitted decisions as a function of AI (O'Doherty et al., 2007). We assumed that RI would be a primary factor contributing to any fitting errors between actual decisions and the estimated model, that is, the MEE (see Materials and Methods). One subject with no again decision and another subject whose decisions were invariant across AI were excluded from this analysis due to their poor model fit.

Positive and negative MEEs indicated decisions biased toward the save and again option, respectively (Fig. 6A). A significant increase of MEEs over relative income confirmed our assumption that MEE reflects the degree of the influence of social comparison on decision at a given trial $\left(F_{(1,19)}=22.17, p=0.0002\right)$. The analysis that used the MEEs as a parametric modulator revealed that MEE was positively correlated with the activity of the vmPFC $(x=16, y=36, z=-6, Z=4.07, p<0.05$, corrected; Fig. $6 B)$ and negatively correlated with that of the right insula $(x=34, y=$ $18, z=14, Z=4.62, p<0.05$, corrected; Fig. $6 C)$. 

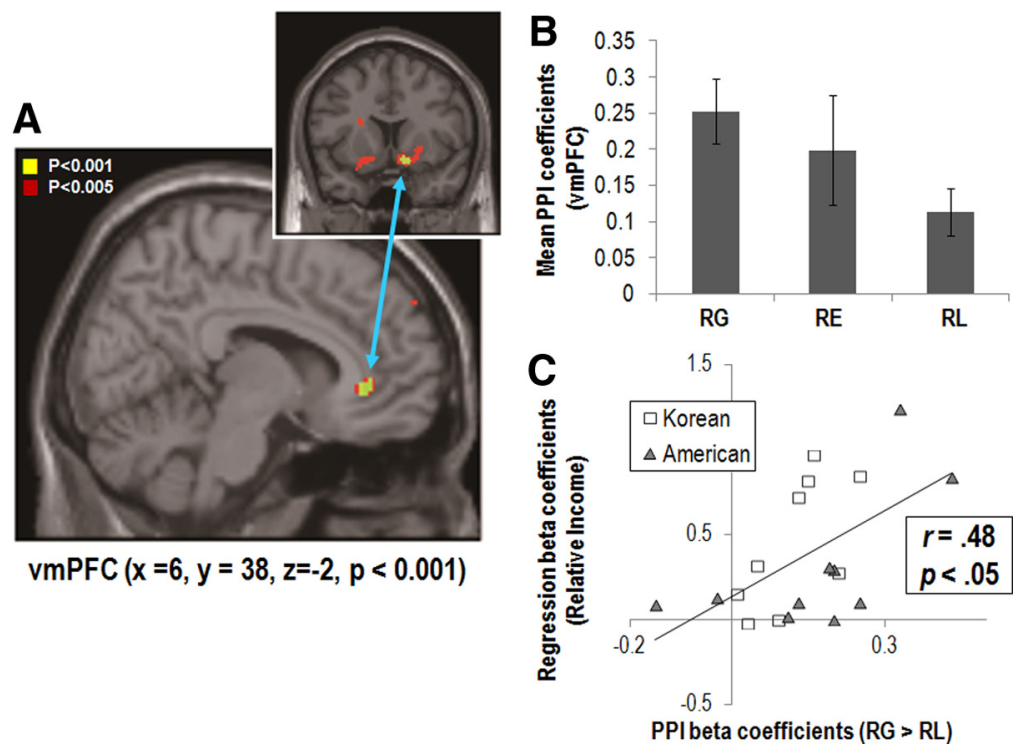

Figure 5. Results from PPI analysis. $A$, The PPI analysis using the VS as the seed point revealed the $\operatorname{vmPFC}(x=6, y=$ $38, z=-2)$ showing functional connectivity with the VS during relative gain versus loss trials. $\boldsymbol{B}$, The average of PPI $\beta$ coefficients for each relative income level. $C$, The $\beta$ estimates of the peak voxel in the cluster of the vmPFC from the PPI interaction map $(A)$ were positively correlated with the $\beta$ coefficients from the logistic regression analysis using the behavioral decision data.

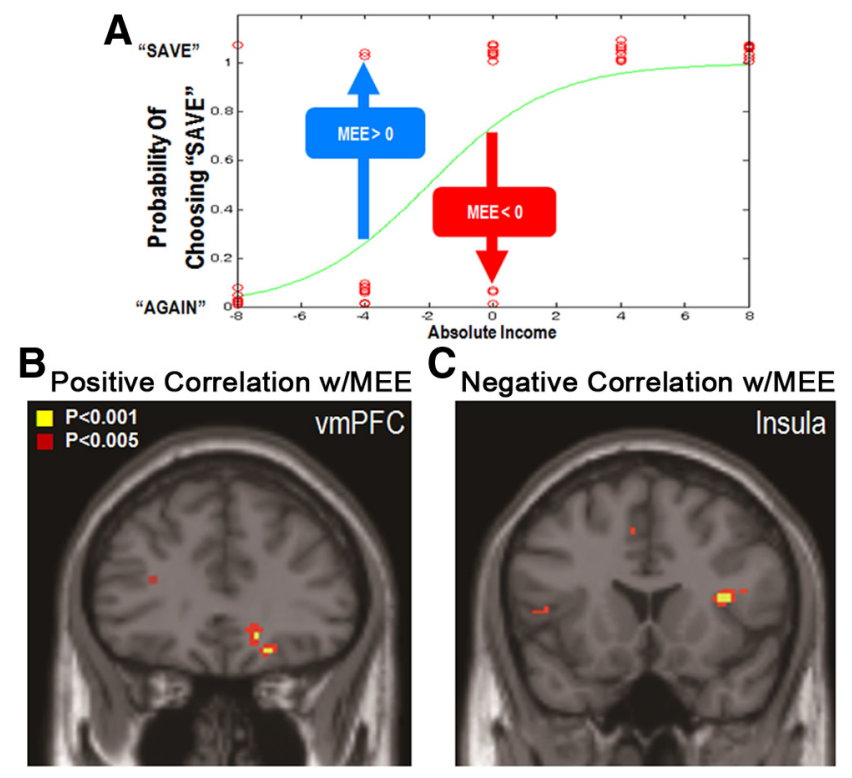

Figure 6. A, MEE computed by taking the distance between the individually estimated probabilities of the save decision increasing as a function of absolute income. Analyses using MEEs as parametric modulators revealed that the $\operatorname{vmPFC}(\boldsymbol{B}: x=16, y=36, z=-6)$ and the right insula $(C: x=34, y=18, z=14)$ showed significant positive and negative correlations with MEE, respectively.

To ensure that MEE does not simply reflect save or again decisions, we divided save and again trials into two subdivisions (i.e., upper $50 \%$ and lower $50 \%$ ) based on the associated MEE parameters using a median split. Thus, a new analysis included four regressors: upper $50 \%$ and lower $50 \%$ save trials, and upper $50 \%$ and lower $50 \%$ again trials. When the parameter estimates were plotted separately for the four regressors, both the vmPFC and the insula showed a linear rather than a step-like trend as a function of MEE (Fig. 7). More specifically, we found significant linear trends for both the vmPFC $\left(F_{(1,15)}=31.90, p=\right.$ $0.00005)$ and the insula $\left(F_{(1,15)}=11.40, p=\right.$ $0.004)$

Then, to identify the cultural differences using MEE, we first converted MEEs into absolute values and summed them across all the trials to obtain mean MEE for each participant. There was a tendency toward higher mean MEEs in the Korean $(\mathrm{M}=0.56, \mathrm{SE}=0.07)$ compared with the American participants $(\mathrm{M}=$ 0.48 , SE $=0.08)$, which was not significant $\left(F_{(1,17)}=0.523, p=0.47\right)$. However, we found a significant correlation between the degree to which participants' decisions were affected by RI (Beta_RI) and MEE, particularly for the relative zero $\left(r_{(19)}=0.49, p=0.034\right)$ and gains $\left(r_{(19)}=0.47, p=0.029\right)$, indicating that MEE indeed reflects trialby-trial decision biases due to relative incomes.

For the neuroimaging results with MEE parameters, we observed neither cultural difference nor correlation with individuals' RI betas in both the medial orbitofrontal cortex and the insula, possibly because the MEE parameters already reflect individual variability in the sensitivity to relative income.

\section{Discussion}

In the present study, we tested the neural substrates of decision bias due to social comparison and its cultural variation between Koreans and Americans. The present study demonstrated that, in a financial gambling task with two players engaged in simultaneous card games, participants' choices were influenced by relative incomes; this was accompanied by changes in activity in reward-sensitive regions of the brain such as the VS and the vmPFC. As predicted, the degree to which participants' decisions and brain activity were affected by relative incomes varied by their cultural membership. Specifically, Koreans were more likely to be affected by another's income than Americans at both the behavioral and neural levels. Further analyses revealed that the VS was functionally connected positively with the vmPFC and negatively with the insular cortex during the relative gain versus loss conditions. The strength of functional connectivity between the VS and the vmPFC predicted individual variability in the degree to which participants' decisions were affected by relative incomes. Finally, additional analysis using a model-based fMRI method revealed that the activities in the vmPFC and insula predicted trial-by-trial fluctuations in positive and negative decision biases, respectively. In summary, the present study provides the first empirical evidence for the neural systems involved in biasing financial decisions due to social comparison, and their individual as well as cultural variations.

As expected, despite the presence of individual variability, most of the participants in the present study were influenced by relative incomes while making their choices in the games. Consistent with previous findings, the Korean participants were more likely to be affected by relative than absolute incomes, whereas the American participants' decisions were influenced by absolute rather than relative incomes. This 

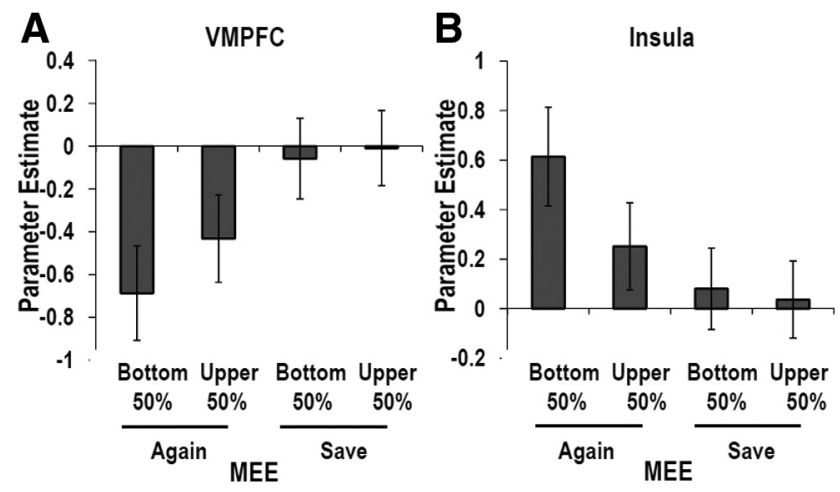

Figure 7. Mean parameter estimates from the $\operatorname{vmPFC}(\boldsymbol{A})$ and the insula $(\boldsymbol{B})$ in Figure 6 divided into upper and lower $50 \%$ MEE trials separately for save and "again" decisions.

provides evidence further supporting the idea that members from interdependent cultures are more likely to be affected by information about others, that is, social comparison, compared with those from independent cultures (Chung and Mallery, 1999; Gibbons and Buunk, 1999; White and Lehman, 2005). It is interesting to note that the participant who showed the biggest influence from relative incomes was American; this perhaps supports a more recent view that both culture and individual differences jointly contribute to determining final behavioral phenotype (Leung and Cohen, 2011).

Consistent with our prediction, the behavioral data in the present study were highly resonant with the activity pattern of the VS, which has been frequently reported as a key region encoding reward in human and animals (Apicella et al., 1991; Schultz et al., 1997; Delgado et al., 2000; Knutson et al., 2001; O'Doherty et al., 2004). It was recently reported that the activity of the VS can be influenced by various contextual factors (Fliessbach et al., 2007; Elliott et al., 2008; Dvash et al., 2010), and seems to encode not only absolute but also relative value of rewards in social situations. More specifically, monetary gains higher than other subjects' led to increased VS activity, compared with gains equal to or lower than those of others (Fliessbach et al., 2007; Dvash et al., 2010). The present study expands previous findings even further by showing that VS activity can be also modulated by the cultural membership of participants, that is, Koreans are more sensitive to relative than absolute incomes and vice versa for Americans. Given the complexity of relative versus absolute income value computation, it is not difficult to hypothesize that VS activity may be modulated by RI possibly through its communication with the prefrontal cortex.

Consistent with the idea of greater interdependency in Eastern cultures (Markus and Kitayama, 1991), the vmPFC also showed a significant social comparison effect only in Koreans. This cultural difference was even greater than that in the VS, perhaps indicating a greater role of the vmPFC in the computation of socially influenced decision value. A large body of literature supports the primary role of the vmPFC in encoding subjective values critical for guiding decisions in various situations (Tanaka et al., 2004; Kim et al., 2006; Kable and Glimcher, 2007; Kim et al., 2007; Chib et al., 2009) and also in encoding relative rather than absolute values (Elliott et al., 2008; Bault et al., 2011; Lim et al., 2011). Strong reciprocal anatomical connections exist between the VS and the vmPFC (Haber et al., 2006; Haber and Knutson, 2010), which are believed to be critical for computing decision values. Consistent with these findings, the vmPFC showed strong functional connectivity with the VS during relative gain versus loss trials, which also predicted individual variability in social comparison. Moreover, vmPFC activity correlated with trial-bytrial deviation of actual decisions from individual decision probability estimated by absolute values. These findings perhaps emphasize the role of the vmPFC in computing value signals for biasing decisions and shaping the neural activity of the VS in social contexts.

We found that right insula activity showed negative correlation with the VS during the relative gain versus loss condition and predicted trial-by-trial biases in decision due to relative incomes. Several previous studies have demonstrated the role of the insula in encoding inequality, revealing increases in its activity following unfair offers from proposers during ultimatum games (Sanfey et al., 2003), unequal distribution of meals to orphans (Hsu et al., 2008), and relatively unequal payment during multiplayer games (Fliessbach et al., 2007; Haruno and Frith, 2010). In addition, we recently observed that functional connectivity between the insula and the orbitofrontal cortex predicted negative decision biases during ultimatum games (H. Kim et al., 2012). The present findings together with previous studies suggest that the insula may bias the decision value computation in the orbitofrontal cortex by signaling emotional salience elicited by unfair or unequal social situations.

Consistent with our prediction based on previous studies of cultural differences, participants' decisions and activity in both the VS and vmPFC were significantly modulated by participants' cultural membership. To our best knowledge, this is the first study documenting cultural differences in social comparison at the neural level. According to a more recent view, however, culture may be important in itself, but individual difference should also not be ignored as both within- and between-culture variations play critical roles in shaping human behavior (Leung and Cohen, 2011). In fact, individual difference in social comparison was predicted more significantly by VS-vmPFC connectivity strength than by cultural membership. Perhaps a more sophisticated understanding of the neural basis of social behaviors will be needed to elucidate the fundamental principles by which individual and cultural factors jointly shape human behavior.

Several issues deserve further discussion concerning the present study. For example, it was recently observed that rewardrelated neural responses were modulated by the relative difference in the participants' endowed income levels of the participants in during a monetary financial distribution task (Tricomi et al., 2010). Unlike the present study, participants' income levels were experimentally manipulated before the main experiment in the study. Given that participants' subjective social status and perceived inequality may well be important factors modulating the social comparison effect, this deserves further investigation in future studies.

Second, all of the American participants were told that they had been recruited based on their nationality and ethnicity, and that they would be playing with another participant recruited with the same advertisement. Therefore, it seems unlikely that the participants would have assumed that their partners were Korean, although, given that the American participants were all foreign visitors, a somewhat different result might be obtained if a similar study was conducted in the United States.

Third, the possibility remains that the cultural and/or individual differences observed in the present study may reflect 
different cognitive information processing styles rather than social comparison. That is, the optimal strategy adopted by those with a global type of thinking appeared to be to accept on all the trials with positive outcomes and reject on all the trials with negative outcomes, in the hope of increasing the probability of drawing more trials with positive outcomes at the end of the game. In contrast, the social comparison effect observed in the present study may be best characterized by a behavioral tendency to focus on local information (i.e., the others' income levels) rather than on a more global aspect of the game structure. In fact, it is an interesting but unexplored hypothesis whether these different cognitive styles may underlie cultural differences in social comparison. Future investigations on this specific issue may further advance our understanding of the social comparison effect.

Finally, several factors other than cultural membership, such as subjective level of happiness (Lyubomirsky and Ross, 1997) and power distance (Guimond et al., 2006), have been shown to affect social comparison. We administered none of the behavioral measures necessary to test these hypotheses, but we hope they will be explored further in future studies.

In summary, this study revealed for the first time the neural basis of individual and cultural variability in the influence of social comparison on decisions, emphasizing the role of the vmPFC in the process. We believe that our findings could serve as a useful framework for future research examining how genetic and environmental factors interact with each other to shape the social comparison effect.

\section{References}

Apicella P, Ljungberg T, Scarnati E, Schultz W (1991) Responses to reward in monkey dorsal and ventral striatum. Exp Brain Res 85:491-500. Medline

Bault N, Joffily M, Rustichini A, Coricelli G (2011) Medial prefrontal cortex and striatum mediate the influence of social comparison on the decision process. Proc Natl Acad Sci U S A 108:16044-16049. CrossRef Medline

Chiao JY, Harada T, Komeda H, Li Z, Mano Y, Saito D, Parrish TB, Sadato N, Iidaka T (2009) Neural basis of individualistic and collectivistic views of self. Hum Brain Mapp 30:2813-2820. CrossRef Medline

Chib VS, Rangel A, Shimojo S, O’Doherty JP (2009) Evidence for a common representation of decision values for dissimilar goods in human ventromedial prefrontal cortex. J Neurosci 29:12315-12320. CrossRef Medline

Chung T, Mallery P (1999) Social comparison, individualism-collectivism, and self-esteem in China and the United States. Curr Psychol 18:340-352. CrossRef

Clark AE, Oswald AJ (1996) Satisfaction and comparison income. J Public Econ 61:359-381. CrossRef

Cox RW (1996) AFNI: software for analysis and visualization of functional magnetic resonance neuroimages. Comput Biomed Res 29:162-173. CrossRef Medline

Deichmann R, Gottfried JA, Hutton C, Turner R (2003) Optimized EPI for fMRI studies of the orbitofrontal cortex. Neuroimage 19:430-441. CrossRef Medline

Delgado MR, Nystrom LE, Fissell C, Noll DC, Fiez JA (2000) Tracking the hemodynamic responses to reward and punishment in the striatum. J Neurophysiol 84:3072-3077. Medline

Di Martino A, Scheres A, Margulies DS, Kelly AM, Uddin LQ, Shehzad Z, Biswal B, Walters JR, Castellanos FX, Milham MP (2008) Functional connectivity of human striatum: a resting state FMRI study. Cereb Cortex 18:2735-2747. CrossRef Medline

Dvash J, Gilam G, Ben-Ze'ev A, Hendler T, Shamay-Tsoory SG (2010) The envious brain: the neural basis of social comparison. Hum Brain Mapp 31:1741-1750. Medline

Elliott R, Agnew Z, Deakin JF (2008) Medial orbitofrontal cortex codes relative rather than absolute value of financial rewards in humans. Eur J Neurosci 27:2213-2218. CrossRef Medline
Festinger L (1954) A theory of social comparison processes. Hum Relations 7:117-140. CrossRef

Fliessbach K, Weber B, Trautner P, Dohmen T, Sunde U, Elger CE, Falk A (2007) Social comparison affects reward-related brain activity in the human ventral striatum. Science 318:1305-1308. CrossRef Medline

Friston KJ, Buechel C, Fink GR, Morris J, Rolls E, Dolan RJ (1997) Psychophysiological and modulatory interactions in neuroimaging. Neuroimage 6:218-229. CrossRef Medline

Gibbons FX, Buunk BP (1999) Individual differences in social comparison: development of a scale of social comparison orientation. J Pers Soc Psychol 76:129-142. CrossRef Medline

Gilbert DT, Giesler RB, Morris KA (1995) When comparisons arise. J Pers Soc Psychol 69:227-236. CrossRef Medline

Gitelman DR, Penny WD, Ashburner J, Friston KJ (2003) Modeling regional and psychophysiologic interactions in fMRI: the importance of hemodynamic deconvolution. Neuroimage 19:200-207. CrossRef Medline

Guimond S, Chatard A, Martinot D, Crisp RJ, Redersdorff S (2006) Social comparison, self-stereotyping, and gender differences in self-construals. J Pers Soc Psychol 90:221-242. CrossRef Medline

Haber SN, Knutson B (2010) The reward circuit: linking primate anatomy and human imaging. Neuropsychopharmacology 35:4-26. CrossRef Medline

Haber SN, Kim KS, Mailly P, Calzavara R (2006) Reward-related cortical inputs define a large striatal region in primates that interface with associative cortical connections, providing a substrate for incentive-based learning. J Neurosci 26:8368-8376. CrossRef Medline

Haruno M, Frith CD (2010) Activity in the amygdala elicited by unfair divisions predicts social value orientation. Nat Neurosci 13:160-161. CrossRef Medline

Hsu M, Anen C, Quartz SR (2008) The right and the good: distributive justice and neural encoding of equity and efficiency. Science 320:10921095. CrossRef Medline

Kable JW, Glimcher PW (2007) The neural correlates of subjective value during intertemporal choice. Nat Neurosci 10:1625-1633. CrossRef Medline

Kennedy DN, Lange N, Makris N, Bates J, Meyer J, Caviness VS Jr (1998) Gyri of the human neocortex: an MRI-based analysis of volume and variance. Cereb Cortex 8:372-384. CrossRef Medline

Kim B, Sung YS, McClure SM (2012) The neural basis of cultural differences in delay discounting. Philos Trans R Soc Lond B Biol Sci 367:650-656. CrossRef Medline

Kim H, Shimojo S, O’Doherty JP (2006) Is avoiding an aversive outcome rewarding? Neural substrates of avoidance learning in the human brain. PLoS Biol 4:e233. CrossRef Medline

Kim H, Adolphs R, O’Doherty JP, Shimojo S (2007) Temporal isolation of neural processes underlying face preference decisions. Proc Natl Acad Sci U S A 104:18253-18258. CrossRef Medline

Kim H, Choi MJ, Jang IJ (2012) Lateral OFC activity predicts decision bias due to first impressions during ultimatum games. J Cogn Neurosci 24: 428-439. CrossRef Medline

Knutson B, Adams CM, Fong GW, Hommer D (2001) Anticipation of increasing monetary reward selectively recruits nucleus accumbens. J Neurosci 21:RC159. Medline

Leung AK, Cohen D (2011) Within- and between-culture variation: individual differences and the cultural logics of honor, face, and dignity cultures. J Pers Soc Psychol 100:507-526. CrossRef Medline

Lim SL, O'Doherty JP, Rangel A (2011) The decision value computations in the vmPFC and striatum use a relative value code that is guided by visual attention. J Neurosci 31:13214-13223. CrossRef Medline

Lyubomirsky S, Ross L (1997) Hedonic consequences of social comparison: a contrast of happy and unhappy people. J Pers Soc Psychol 73:11411157. CrossRef Medline

Markus HR, Kitayama S (1991) Culture and the Self: implications for cognition, emotion, and motivation. Psychol Rev 98:224-253. CrossRef

Nisbett RE, Peng K, Choi I, Norenzayan A (2001) Culture and systems of thought: holistic versus analytic cognition. Psychol Rev 108:291-310. CrossRef Medline

O'Doherty JP, Hampton A, Kim H (2007) Model-based fMRI and its application to reward learning and decision making. Ann N Y Acad Sci 1104: 35-53. CrossRef Medline

O’Doherty J, Dayan P, Schultz J, Deichmann R, Friston K, Dolan RJ (2004) 
Dissociable roles of ventral and dorsal striatum in instrumental conditioning. Science 304:452-454. CrossRef Medline

Sanfey AG, Rilling JK, Aronson JA, Nystrom LE, Cohen JD (2003) The neural basis of economic decision-making in the Ultimatum Game. Science 300:1755-1758. CrossRef Medline

Schultz W, Dayan P, Montague PR (1997) A neural substrate of prediction and reward. Science 275:1593-1599. CrossRef Medline

Takahashi H, Kato M, Matsuura M, Mobbs D, Suhara T, Okubo Y (2009) When your gain is my pain and your pain is my gain: neural correlates of envy and schadenfreude. Science 323:937-939. CrossRef Medline

Tanaka SC, Doya K, Okada G, Ueda K, Okamoto Y, Yamawaki S (2004) Prediction of immediate and future rewards differentially recruits cortico-basal ganglia loops. Nat Neurosci 7:887-893. CrossRef Medline

Tenbrunsel AE, Diekmann KA (2002) Job-decision inconsistencies involving social comparison information: the role of dominating alternatives. J Appl Psychol 87:1149-1158. CrossRef Medline

Tricomi E, Rangel A, Camerer CF, O’Doherty JP (2010) Neural evidence for inequality-averse social preferences. Nature 463:1089-1091. CrossRef Medline

White K, Lehman DR (2005) Culture and social comparison seeking: the role of self-motives. Pers Soc Psychol Bull 31:232-242. CrossRef Medline

Zhu Y, Zhang L, Fan J, Han S (2007) Neural basis of cultural influence on self-representation. Neuroimage 34:1310-1316. CrossRef Medline 